\title{
A Fast MoM Calculation Technique Using Sinusoidal Basis and Testing Functions for a Wire on a Dielectric Substrate and Its Application to Meander Loop and Grid Array Antennas
}

\author{
Hisamatsu Nakano, Fellow, IEEE, Toru Kawano, Member, IEEE, Yousuke Kozono, and Junji Yamauchi, Member, IEEE
}

\begin{abstract}
The input impedance matrix element of the method of moments (MoM) for an arbitrarily shaped wire antenna printed on a dielectric material $Z_{m, n}$ is formulated to be composed of three terms $Z_{m, n}^{\psi s}, Z_{m, n}^{\psi}$, and $\Delta Z_{m, n}$ involving single-, double-, and triple-integral calculations, respectively. The MoM based on the $Z_{m, n}$ formulated in this paper (new MoM) is applied to two antennas-a meander loop antenna and a grid array antenna-as well as a simple loop used as a reference antenna. The computation time to obtain the current distribution of each antenna by the new MoM technique is compared with the time required for the conventional MoM, which has an impedance matrix element composed of four terms, all involving triple-integral calculations. It is revealed that the new MoM drastically reduces the computation time: for example, by a factor of 937 for the grid array antenna. In addition, the radiation characteristics of these two antennas are discussed. It is found that a reduced-size meander loop (62\% smaller than the simple loop reference) has a radiation pattern similar to the simple loop reference. It is also found that the grid array has an axial beam radiation pattern without side lobes in the principal planes.
\end{abstract}

Index Terms-Grid array antenna, meander loop antenna, method of moments (MoM), printed wire.

\section{INTRODUCTION}

A $\mathrm{N}$ integral equation for a straight wire printed on a dielectric substrate has been derived and applied to the analysis of a printed dipole antenna [1]. In addition, an integral equation for an arbitrarily shaped wire antenna printed on a dielectric substrate has been derived and applied to the analysis of printed zigzag dipole, loop, and spiral antennas [2]. Note that the dielectric substrate used in [1] and [2] is backed by a conducting plane, and the antenna structure is referred to as the C-type structure in this paper. Also, note that the integral equation for the C-type structure (referred to as the C-type integral equation) has been solved using the method of moments (MoM) [3].

The MoM impedance matrix element $Z_{m, n}$ for the C-type integral equation in [2] is explained in detail in [4]. This matrix element is composed of four terms $g_{m-1, n-1}, g_{m-1, n}, g_{m, n-1}$, and $g_{m, n}$, all being expressed in triple-integral form and requiring a long time to compute.

Manuscript received April 16, 2004; revised December 25, 2004.

H. Nakano, Y. Kozono, and J. Yamauchi are with the College of Engineering, Hosei University, Tokyo 184-8584, Japan (e-mail: nakano@k.hosei.ac.jp).

T. Kawano is with the Communication Engineering Department, National Defense Academy, Kanagawa 239-8686, Japan.

Digital Object Identifier 10.1109/TAP.2005.856314
An integral equation for an arbitrarily shaped wire antenna printed on a semi-infinite dielectric material has already been derived in [5]. The antenna structure in [5] is referred to as the D-type structure in this paper. It is emphasized that the D-type structure does not have a conducting plane backing the dielectric, unlike the C-type structure. Note that the integral equation for the D-type structure (referred to as the D-type integral equation) is formulated to have the same form as that for the C-type integral equation; however, the Hertz vector potential functions appearing in the D-type integral equation differ from those in the C-type integral equation.

The D-type integral equation has also been solved using the MoM [5], where the impedance matrix element $Z_{m, n}$ is expressed as $Z_{m, n}=Z_{m, n}^{\rho}+\Delta Z_{m, n}$. The first term $Z_{m, n}^{\rho}$ is composed of four double-integral terms $\left(g_{m-1, n-1}^{\rho}, g_{m-1, n}^{\rho, n}\right.$, $g_{m, n-1}^{\rho}$, and $\left.g_{m, n}^{\rho}\right)$. The second term $\Delta Z_{m, n}$ is composed of four triple-integral terms $\left(\Delta g_{m-1, n-1}, \Delta g_{m-1, n}, \Delta g_{m, n-1}\right.$, and $\left.\Delta g_{m, n}\right)$. The second term smoothly varies with the distance between source and observation points and is calculated using an interpolation technique. This interpolation technique reduces the computation time.

This paper presents a newly developed MoM technique for solving the C-type integral equation, with the aim of reducing the long computation time. The technique comprises application of the interpolation technique in [5] and use of a new technique for reducing the computation time of the double-integral calculations in the MoM impedance matrix element. In addition, the radiation characteristics of two antennas obtained using the newly developed MoM technique are presented.

A decomposition technique for the Hertz vector potential functions (used for the new MoM calculation) is described in Section II. The decomposition technique results in the impedance matrix element $Z_{m, n}=Z_{m, n}^{C}+\Delta Z_{m, n}^{C}$. The first term $Z_{m, n}^{C}$ involves double-integral calculations. If a single-integral term is extracted from the first double-integral term $Z_{m, n}^{C}$, that is

$$
\begin{aligned}
& Z_{m, n \mid \text { double-integral }}^{C} \\
& \quad=Z_{m, n \mid \text { single-integral }}^{C}+Z_{m, n \mid \text { remaining double-integral }}^{C}
\end{aligned}
$$

the remaining double-integral calculations for the $Z_{m, n}^{C}$ after the extraction are expected to take less computation time than the original double-integral calculations for the $Z_{m, n}^{C}$ (such an extraction is discussed in [6]; however, it is not described in detail). 
Based on this expectation, this paper performs extraction of a single-integral term (realized on the basis of a closed-form electric field expression, which is not presented in [5] and [6]) from the original double-integral term $Z_{m, n}^{C}$. Note that the second term $\Delta Z_{m, n}^{C}$ in the $Z_{m, n}$ involves triple-integral calculations and is processed using the interpolation technique in [5] to reduce computation time.

Application examples of the newly developed MoM impedance matrix element $Z_{m, n}$ to printed antennas are shown in Section III, where meander loop and grid array antennas [7]-[11] are analyzed and reduction in the computation time is discussed. The computation time to obtain the current distribution of each antenna is compared with the time required using the conventional $Z_{m, n}$ in [2]. It is found that the computation time for the grid array antenna, for example, is drastically reduced by a factor of 937 .

In addition to demonstrating the reduction in the computation time, this paper analyzes the radiation characteristics of these two antennas. The analysis reveals that transformation from the straight-arm filaments of the loop to meandering arm filaments contributes to reduction in the loop size, while not deteriorating the radiation pattern. It is also revealed that the grid array antenna radiates an axial beam without side lobes in the two principal planes. Note that the validity of the MoM analysis results based on the new $Z_{m, n}$ is confirmed by comparing them with results obtained using the finite-difference time-domain method (FDTDM) [12].

Section IV summarizes the results obtained in this paper. It is emphasized that two processes lead to a remarkable reduction in computation time: application of the interpolation technique shown in [5] to the triple-integral term $\Delta Z_{m, n}^{C}$ and the new extraction of a single-integral term of $Z_{m, n \mid \text { single-integral }}^{C}\left(=Z_{m, n}^{\psi s}\right)$ from the original double-integral $Z_{m, n}^{C}$.

\section{NUMERICAL METHOD}

\section{A. Integral Equation for a Wire on a Dielectric Substrate}

Fig. 1 shows an arbitrarily shaped wire on a dielectric substrate backed by a conducting plane, where both the substrate (which has relative permittivity $\varepsilon_{r}$ and thickness $B$ ) and the conducting plane are of infinite extent. It is assumed that the dielectric substrate is lossless, and the wire is perfectly conducting. It is also assumed that the wire radius is small relative to the wavelength and only the axial component of the current contributes to the antenna characteristics. The electric field tangential to the wire axis (scattering field) $E_{s}(s)$ is expressed as

$E_{s}(s)=\int_{0}^{L}\left[\frac{\partial}{\partial s}\left(-\frac{\partial \Pi^{s}}{\partial s^{\prime}}+\frac{\partial \Pi}{\partial s^{\prime}}\right)+k_{0}^{2} \Pi^{s}\left(\hat{s}^{\prime} \bullet \hat{s}\right)\right] I\left(s^{\prime}\right) d s^{\prime}$

where $s[=s(x, y)]$ and $s^{\prime}\left[=s^{\prime}\left(x^{\prime}, y^{\prime}\right)\right]$ are the distances along the wire from its starting point to observation and source points, respectively; $\hat{s}$ and $\hat{s}^{\prime}$ are the unit vectors tangential to the wire axis at distances $s$ and $s^{\prime}$, respectively; $I\left(s^{\prime}\right)$ is the current at distance $s^{\prime} ; L$ is the wire length; $k_{0}$ is the wave number in free

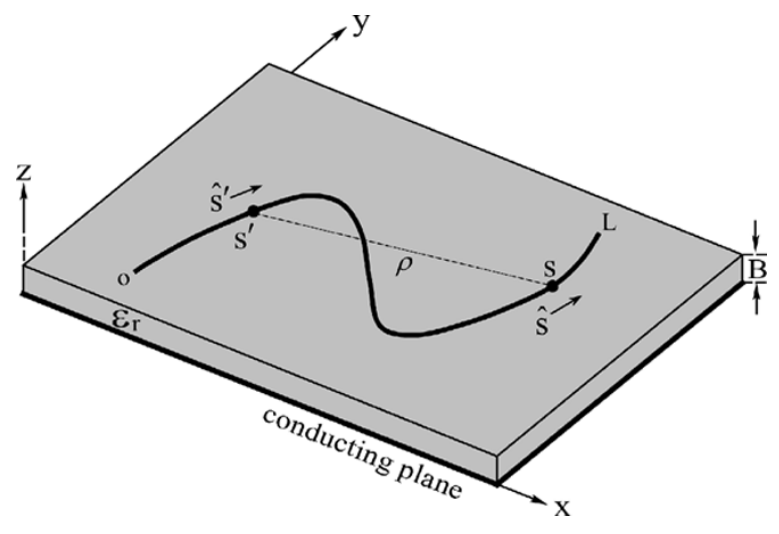

Fig. 1. An arbitrarily shaped wire on a dielectric substrate.

space $\left(\omega \sqrt{\mu_{0} \varepsilon_{0}}\right)$; and $\Pi^{s}\left(s, s^{\prime}\right)$ and $\Pi\left(s, s^{\prime}\right)$ are components of the Hertz vector potential function [1]

$$
\begin{aligned}
& \Pi^{s}\left(s, s^{\prime}\right) \\
& =2 q \lim _{z \rightarrow B} \int_{0}^{\infty} J_{0}(\lambda \rho) e^{-u_{0}(z-B)} \frac{\lambda}{D_{e}(\lambda)} d \lambda \\
& \Pi\left(s, s^{\prime}\right) \\
& =2 q\left(\varepsilon_{r}-1\right) \lim _{z \rightarrow B} \int_{0}^{\infty} J_{0}(\lambda \rho) e^{-u_{0}(z-B)} \frac{u_{0} \lambda}{D_{e}(\lambda) D_{m}(\lambda)} d \lambda
\end{aligned}
$$

where $q=-j /\left(4 \pi \omega \varepsilon_{0}\right) ; J_{0}(\lambda \rho)$ is a Bessel function of the first kind of order zero with $\rho=\rho\left(s, s^{\prime}\right)=\left[\left(x-x^{\prime}\right)^{2}+\left(y-y^{\prime}\right)^{2}\right]^{1 / 2}$; $D_{e}(\lambda)=u_{0}+u_{e} \operatorname{coth} u_{e} B$; and $D_{m}(\lambda)=u_{0} \varepsilon_{r}+u_{e} \tanh u_{e} B$ with $u_{0}=\sqrt{\lambda^{2}-k_{0}^{2}}$ and $u_{e}=\sqrt{\lambda^{2}-\varepsilon_{r} k_{0}^{2}}$.

Equation (1) is referred to as the C-type integral equation. This has the same form as that of the D-type integral equation [5] but $\Pi^{s}\left(s, s^{\prime}\right)$ and $\Pi\left(s, s^{\prime}\right)$ of the C-type integral equation differ from those of the D-type integral equation. The conventional MoM impedance matrix element $Z_{m, n}$ in [2] for the C-type integral equation is composed of four terms $\left(g_{m-1, n-1}, g_{m-1, n}\right.$, $g_{m, n-1}$, and $\left.g_{m, n}\right)$, each involving triple-integral calculations, and hence it requires considerable computation time. Reduction in the computation time in this paper is performed through two steps. The first step is to decompose each of $\Pi^{s}\left(s, s^{\prime}\right)$ and $\Pi\left(s, s^{\prime}\right)$ into a free-space Green's function term (or a weighted free-space Green's function term) and a remaining term; in other words, the first step is to derive a perturbation term, from which the triple-integral calculations in the MoM impedance matrix element are formulated. Note that the interpolation technique in [5] is applied to the triple-integral calculations. The second step is to decompose an original double-integral term of the MoM impedance matrix element into a single-integral term and a remaining term. It is emphasized that the second step provides a new technique of reducing the computation time required for the original double-integral term.

\section{B. Decomposition of the Scattering Field $E_{s}(s)$}

The first step in reducing the computation time is to derive a perturbation term. Subtracting a free-space Green's function $\psi^{s}$ 
$\left(=q \lim _{z \rightarrow B} e^{-j k_{0} R} / R\right.$ with $\left.R=\left[\rho^{2}+(z-B)^{2}\right]^{1 / 2}\right)$ from $\Pi^{s}$ yields the perturbation term $\Delta \psi^{s}$ [13]

$$
\begin{aligned}
\Delta \psi^{s} & =\Pi^{s}-\psi^{s} \\
& =q \lim _{z \rightarrow B} \int_{0}^{\infty} J_{0}(\rho \lambda) e^{-u_{0}(z-B)} \frac{\lambda}{u_{0}}\left[\frac{2 u_{0}}{D_{e}(\lambda)}-1\right] d \lambda .
\end{aligned}
$$

Similarly, subtracting a weighted Green's function $\tau \psi^{s}$ (defined as $\psi$ ) from $\Pi$ yields the perturbation term $\Delta \psi$

$$
\begin{aligned}
\Delta \psi & =\Pi-\psi \\
& =q \lim _{z \rightarrow B} \int_{0}^{\infty} J_{0}(\rho \lambda) e^{-u_{0}(z-B)} \frac{\lambda}{u_{0}}\left[\frac{2\left(\varepsilon_{r}-1\right) u_{0}^{2}}{D_{m}(\lambda) D_{e}(\lambda)}-\tau\right] d \lambda
\end{aligned}
$$

where

$$
\tau=\frac{\varepsilon_{r}-1}{\varepsilon_{r}+1} .
$$

Using (4) and (5), (1) is decomposed into

$$
\begin{aligned}
E_{s}(s) & =\left(E_{\psi s}+E_{\psi}\right)+\Delta E \\
& =-E_{\tan }^{i}(s)
\end{aligned}
$$

where $E_{\tan }^{i}(s)$ is the tangential component of an incident electric field and

$$
\begin{aligned}
E_{\psi s} & =\int_{0}^{L}\left[-\frac{\partial}{\partial s} \frac{\partial \psi^{s}}{\partial s^{\prime}}+k_{0}^{2} \psi^{s}\left(\hat{s}^{\prime} \bullet \hat{s}\right)\right] I\left(s^{\prime}\right) d s^{\prime} \\
E_{\psi} & =\int_{0}^{L} \frac{\partial}{\partial s} \frac{\partial \psi}{\partial s^{\prime}} I\left(s^{\prime}\right) d s^{\prime} \\
\Delta E & =\int_{0}^{L}\left[\frac{\partial}{\partial s} \frac{\partial\left(-\Delta \psi^{s}+\Delta \psi\right)}{\partial s^{\prime}}+k_{0}^{2} \Delta \psi^{s}\left(\hat{s}^{\prime} \bullet \hat{s}\right)\right] I\left(s^{\prime}\right) d s^{\prime}
\end{aligned}
$$

The sum $\left(E_{\psi s}+E_{\psi}\right)$ in (7) involves single-integral calculations, as seen from (8) and (9), while $\Delta E$ in (7) [or (10)] involves double-integral calculations because each of the perturbation terms $\Delta \psi^{s}$ and $\Delta \psi$ in (10) is expressed in single-integral form, as seen from (4) and (5). When the MoM is applied to (7), the impedance matrix element for $n$th basis and $m$ th testing functions is written as $Z_{m, n}=Z_{m, n}^{C}\left(E_{\psi s}+E_{\psi}\right)+\Delta Z_{m, n}^{C}(\Delta E)$, where the first term $Z_{m, n}^{C}\left(E_{\psi s}+E_{\psi}\right)$ involves double-integral calculations, due to the integration over the single-integral calculations of $E_{\psi s}+E_{\psi}$, and the second term $\Delta Z_{m, n}^{C}(\Delta E)$ involves triple-integral calculations, due to the integration over the double-integral calculations of $\Delta E$. It is noted that the interpolation technique in [5] can be applied to the second term $\Delta Z_{m, n}^{C}(\Delta E)$ to reduce the computation time; therefore, further reduction in the computation time for the impedance matrix element $Z_{m, n}$ depends on the first double-integral term $Z_{m, n}^{C}\left(E_{\psi s}+E_{\psi}\right)$.

The computation labor of the double-integral term $Z_{m, n}^{C}\left(E_{\psi s}+E_{\psi}\right)$ is reduced when $Z_{m, n}^{C}\left(E_{\psi s}+E_{\psi}\right)$ is expressed as the sum of a single-integral term and a remaining double-integral term

$Z_{m, n}^{C}\left(E_{\psi s}+E_{\psi}\right)_{\mid \text {double-integral }}$

$=Z_{m, n}^{C}\left(E_{\psi s}\right)_{\mid \text {single-integral }}+Z_{m, n}^{C}\left(E_{\psi}\right)_{\mid \text {remaining double-integral }}$.

For this, the single-integral $E_{\psi s}$ in $Z_{m, n}^{C}\left(E_{\psi s}\right)$ must be expressed in closed form. This is summarized in the next section.

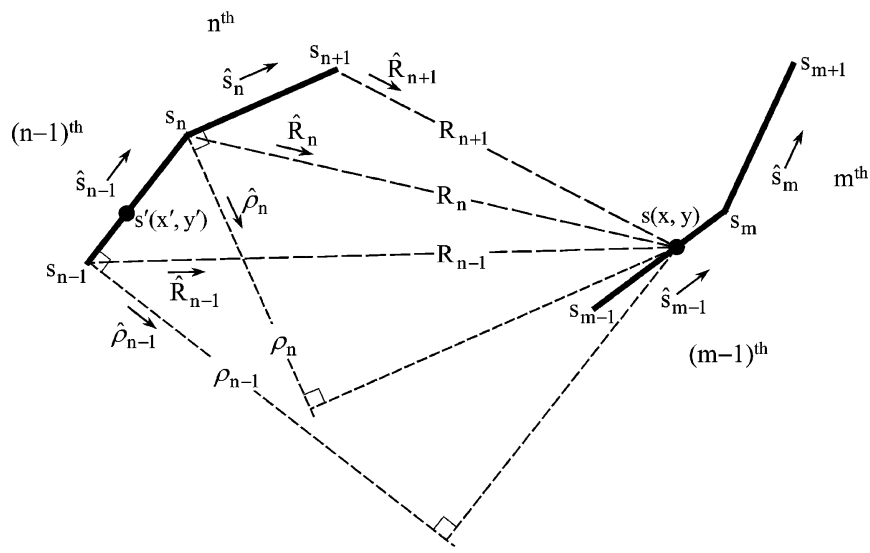

Fig. 2. A magnified view of two pairs of segments.

Note that the closed form of $E_{\psi s}$ derived for the C-type integral equation can also be used for the MoM for the D-type integral equation [5].

\section{Closed Form of $E_{\psi s}$}

The second step in reducing the computation time starts with subdividing the wire shown in Fig. 1 into numerous segments, each being regarded as linear. Fig. 2 shows a magnified view of two pairs of segments in the vicinity of $s^{\prime}$ and $s$ : one is composed of the $(n-1)$ th and $n$th segments and the other is composed of the $(m-1)$ th and $m$ th segments. The $(n-1)$ th segment starts at $s^{\prime}=s_{n-1}$ and ends at $s^{\prime}=s_{n}$. Similarly, the $(m-1)$ th segment starts at $s=s_{m-1}$ and ends at $s=s_{m}$.

The unknown current on the wire is expanded as $I\left(s^{\prime}\right)=$ $\sum_{n} I_{n} J_{n}\left(s^{\prime}\right)$, where $J_{n}\left(s^{\prime}\right)$ is the $n$th piecewise sinusoidal basis function over a region ranging from $s^{\prime}=s_{n-1}$ to $s^{\prime}=s_{n+1}$; that is

$$
\begin{aligned}
J_{n}\left(s^{\prime}\right) & =\frac{\sin k_{0}\left(s^{\prime}-s_{n-1}\right)}{\sin k_{0}\left(s_{n}-s_{n-1}\right)} \quad \text { for } \quad s_{n-1} \leq s^{\prime} \leq s_{n} \\
& =\frac{\sin k_{0}\left(s_{n+1}-s^{\prime}\right)}{\sin k_{0}\left(s_{n+1}-s_{n}\right)} \quad \text { for } \quad s_{n} \leq s^{\prime} \leq s_{n+1} .
\end{aligned}
$$

Then, (8) is expressed in closed form as

$$
E_{\psi s}=I_{n} c_{\psi s(n)}
$$

where

$$
c_{\psi s(n)}=-\frac{j 30}{\sin k_{0} d}\left[\left(\frac{\mathbf{v}_{n-1}}{\rho_{n-1}}+\frac{\mathbf{v}_{n}}{\rho_{n}}\right)-\left(\mathbf{f}_{n-1}+\mathbf{f}_{n}\right)\right] \bullet \hat{s}
$$

in which the segment lengths are made to be equal $\left(s_{n}-s_{n-1}=\right.$ $\left.s_{n+1}-s_{n}=d\right) ; \rho_{j}(j=n-1, n)$ is the radial distance from the $j$ th wire segment axis to the observation point specified by distance $s(x, y)$, as shown in Fig. 2 ; and $\mathbf{v}_{n-1}, \mathbf{v}_{n}, \mathbf{f}_{n-1}$, and $\mathbf{f}_{n}$ are vector quantities [14]

$$
\begin{aligned}
\mathbf{v}_{n-1}= & {\left[\left(\hat{s}_{n-1} \bullet \hat{R}_{n}\right) e^{-j k_{0} R_{n}} \cos k_{0} d\right.} \\
& \left.-\left(\hat{s}_{n-1} \bullet \hat{R}_{n-1}\right) e^{-j k_{0} R_{n-1}}-j e^{-j k_{0} R_{n}} \sin k_{0} d\right] \hat{\rho}_{n-1} \\
\mathbf{v}_{n}= & {\left[\left(\hat{s}_{n} \bullet \hat{R}_{n}\right) e^{-j k_{0} R_{n}} \cos k_{0} d-\left(\hat{s}_{n} \bullet \hat{R}_{n+1}\right) e^{-j k_{0} R_{n+1}}\right.} \\
& \left.+j e^{-j k_{0} R_{n}} \sin k_{0} d\right] \hat{\rho}_{n}
\end{aligned}
$$




$$
\begin{aligned}
\mathbf{f}_{n-1} & =\left[\cos k_{0} d \frac{e^{-j k_{0} R_{n}}}{R_{n}}-\frac{e^{-j k_{0} R_{n-1}}}{R_{n-1}}\right] \hat{s}_{n-1} \\
\mathbf{f}_{n} & =\left[\cos k_{0} d \frac{e^{-j k_{0} R_{n}}}{R_{n}}-\frac{e^{-j k_{0} R_{n+1}}}{R_{n+1}}\right] \hat{s}_{n}
\end{aligned}
$$

where $R_{j}(j=n-1, n, n+1)$ is the distance from the segment end point [specified by the distance $s_{j}(j=n-1, n, n+1)$ ] to the observation point, as shown in Fig. $2 ; \hat{R}_{j}(j=n-1, n, n+1)$ and $\hat{\rho}_{j}(j=n-1, n)$ are the unit vectors in the $R_{j}$ and $\rho_{j}$ directions, respectively; and $\hat{s}_{j}(j=n-1, n)$ is the unit vector parallel to the $j$ th wire segment axis.

Note that, at this stage, the electric fields $E_{\psi s}, E_{\psi}$, and $\Delta E$ [the components of (7)] are expressed in closed form, singleintegral form, and double-integral form, respectively.

\section{Impedance Matrix Element $Z_{m, n}$}

After the current $I\left(s^{\prime}\right)$ in (9) and (10) is expanded using the $n$th basis function, the $m$ th piecewise sinusoidal testing function $W_{m}(s)=J_{m}(s)$ is applied to (9), (10), and (13) to derive an impedance matrix element $Z_{m, n}$

$$
\begin{aligned}
Z_{m, n}= & \left.m \text { th testing of }\left[\left(E_{\psi s}+E_{\psi}\right)+\Delta E\right)\right]_{n \text {th basis function }} \\
\equiv & {\left[Z_{m, n}^{C}\left(E_{\psi s}+E_{\psi}\right)\right]+\Delta Z_{m, n}^{C}(\Delta E) } \\
= & {\left[Z_{m, n}^{C}\left(E_{\psi s}\right)_{\mid \text {single-integral }}\right.} \\
& \left.+Z_{m, n}^{C}\left(E_{\psi}\right)_{\mid \text {remaining double-integral }}\right]+\Delta Z_{m, n}^{C}(\Delta E) \\
\equiv & {\left[Z_{m, n}^{\psi s}\left(E_{\psi s}\right)+Z_{m, n}^{\psi}\left(E_{\psi}\right)\right]+\Delta Z_{m, n}^{C}(\Delta E) }
\end{aligned}
$$

where $Z_{m, n}^{\psi s}\left(E_{\psi s}\right)$ and $Z_{m, n}^{\psi}\left(E_{\psi}\right)$ are terms based on the electric fields $E_{\psi s}$ [(13)] and $E_{\psi}$ [(9)], respectively, and $\Delta Z_{m, n}^{C}(\Delta E)$ is based on the perturbation electric field $\Delta E$ [(10)]

$$
\begin{aligned}
Z_{m, n}^{\psi s}\left(E_{\psi s}\right)= & \frac{1}{\sin k_{0} d} \int_{s_{m-1}}^{s_{m+1}} c_{\psi s(n)} \sin k_{0}\left(d-\left|s-s_{m}\right|\right) d s \\
Z_{m, n}^{\psi}\left(E_{\psi}\right)= & \left(\frac{k_{0}}{\sin k_{0} d}\right)^{2}\left(c_{m-1, n-1}^{\psi}-c_{m-1, n}^{\psi}-c_{m, n-1}^{\psi}+c_{m, n}^{\psi}\right) \\
\Delta Z_{m, n}^{C}(\Delta E)= & \left(\frac{k_{0}}{\sin k_{0} d}\right)^{2}\left\{\left(\Delta c_{m-1, n-1}+\Delta h_{m-1, n-1}\right)\right. \\
& -\left(\Delta c_{m-1, n}-\Delta h_{m-1, n}\right)-\left(\Delta c_{m, n-1}-\Delta h_{m, n-1}\right) \\
& \left.+\left(\Delta c_{m, n}+\Delta h_{m, n}\right)\right\}
\end{aligned}
$$

in which (23)-(25) are as shown at the bottom of the page.
Note that $\psi_{i, j}, \Delta \psi_{i, j}$, and $\Delta \psi_{i, j}^{s}$ are $\psi, \Delta \psi$, and $\Delta \psi^{s}$, respectively, and the subscripts $i(=m-1, m)$ and $j(=n-1, n)$ of $\psi_{i, j}, \Delta \psi_{i, j}$, and $\Delta \psi_{i, j}^{s}$ indicate that the observation and source points are located on the $i$ th and $j$ th elements, respectively.

$E_{\psi s}$ is formulated in closed form, as shown in (13), and hence $Z_{m, n}^{\psi s}$ in (20) merely involves a single-integral calculation. Also, note that $Z_{m, n}^{\psi}$ in (21) involves double-integral calculations, as seen from $c_{i, j}^{\psi}$ in (23). Only $\Delta Z_{m, n}^{C}$ in (22) involves triple-integral calculations. This is because $\Delta c_{i, j}$ [which is based on $\Delta \psi_{i, j}^{s}$ and $\Delta \psi_{i, j}$, as seen from (24)] and $\Delta h_{i, j}$ [which is based on $\Delta \psi_{i, j}^{s}$, as seen from (25)] involve triple-integral calculations, due to the fact that $\Delta \psi_{i, j}^{s}$ and $\Delta \psi_{i, j}$ have single integral forms, as shown in (4) and (5), respectively.

Thus, the conventional MoM impedance matrix element $Z_{m, n}$ in [2] and [4], composed of four terms $g_{m-1, n-1}$, $g_{m-1, n}, g_{m, n-1}$, and $g_{m, n}$, all involving triple-integral calculations, is simplified to a new impedance matrix element composed of three terms $Z_{m, n}^{\psi s}, Z_{m, n}^{\psi}$, and $\Delta Z_{m, n}^{C}$, involving single-, double-, and triple-integral calculations, respectively. The extraction of the single-integral term $Z_{m, n}^{\psi s}$ from the original double-integral $Z_{m, n}^{C}\left(E_{\psi s}+E_{\psi}\right)$ and application of the interpolation technique in [5] to $\Delta Z_{m, n}^{C}$ lead to a remarkable reduction in computation time, as seen in the following sections.

\section{ANTENNA ANALYSIS}

\section{A. Loop Antenna}

Fig. 3(a) and (b) shows loop antennas, each printed on a dielectric substrate backed by a conducting plane. The substrate (of relative permittivity $\varepsilon_{r}$ and thickness $B$ ) and the conducting plane are of infinite extent. These antennas are referred to as the reference square loop antenna and the meander loop antenna, respectively. Fig. 4 shows the relationship between the sizes of these two loops. The reduction ratio of the meander loop to the reference loop is defined as $r_{\text {loop }}=\left(s_{\text {mnd }} / s_{\mathrm{str}}\right)^{2}=$ $\left(n s_{v} / s_{\text {str }}\right)^{2}$, where $s_{\text {mnd }}$ and $s_{\text {str }}$ are the side lengths of the meander and reference loops, respectively, and $n$ is an integer. Note that $s_{v}$ and $s_{h}$ are the side lengths of the meander cell.

The relative permittivity $\varepsilon_{r}$ and thickness $B$ are chosen to be $\varepsilon_{r}=2.5$ and $B=24 \Delta_{\text {loop }} \approx 0.121 \lambda_{12}$, where $\Delta_{\text {loop }}=0.00505 \lambda_{12}$ with $\lambda_{12}$ being the free-space wavelength at a test frequency of $12 \mathrm{GHz}$. The loop is made of a thin wire of radius $\rho=0.17 \Delta_{\text {loop }} \approx 0.000859 \lambda_{12}$. To form an axial beam (the maximum field is in the $z$ direction), the peripheral length of the reference square loop is chosen to be approximately one guide wavelength $\left(\lambda_{g}\right)$ :

$$
\begin{aligned}
c_{i, j}^{\psi} & =\int_{s_{i}}^{s_{i+1}} \cos k_{0}\left(d-\left|s-s_{m}\right|\right)\left\{\int_{s_{j}}^{s_{j+1}} \psi_{i, j} \cos k_{0}\left(d-\left|s^{\prime}-s_{n}\right|\right) d s^{\prime}\right\} d s \\
\Delta c_{i, j} & =\int_{s_{i}}^{s_{i+1}} \cos k_{0}\left(d-\left|s-s_{m}\right|\right)\left\{\int_{s_{j}}^{s_{j+1}}\left(-\Delta \psi_{i, j}^{s}+\Delta \psi_{i, j}\right) \cos k_{0}\left(d-\left|s^{\prime}-s_{n}\right|\right) d s^{\prime}\right\} d s \\
\Delta h_{i, j} & =\left(\hat{s}_{i} \bullet \hat{s}_{j}\right) \int_{s_{i}}^{s_{i+1}} \sin k_{0}\left(d-\left|s-s_{m}\right|\right)\left\{\int_{s_{j}}^{s_{j+1}} \Delta \psi_{i, j}^{s} \sin k_{0}\left(d-\left|s^{\prime}-s_{n}\right|\right) d s^{\prime}\right\} d s
\end{aligned}
$$




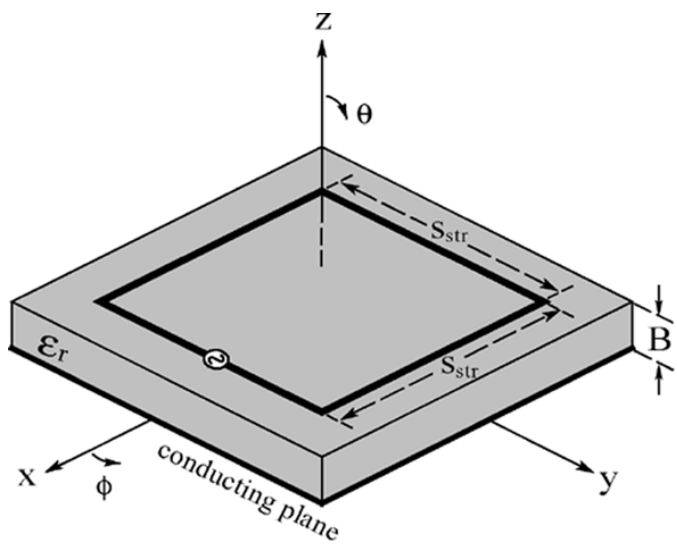

(a)

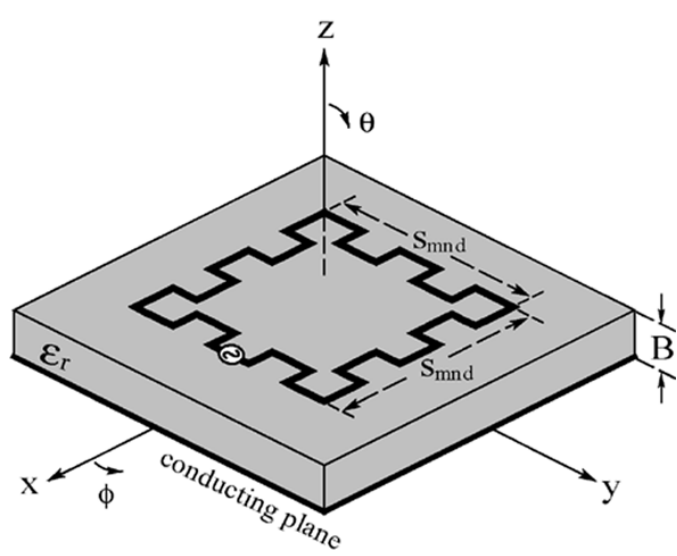

(b)

Fig. 3. Loop antennas. (a) Reference loop antenna and (b) meander loop antenna.

$4 s_{\text {str }}=152 \Delta_{\text {loop }} \approx 1.015 \lambda_{g}$, where the guide wavelength is approximated with $\lambda_{g}=\lambda_{12} / \sqrt{\left(\varepsilon_{r}+1\right) / 2}$. This approximation for the guide wavelength $\lambda_{g}$ is used throughout this paper.

Fig. 5 shows the current distribution $\left(I=I_{r}+j I_{i}\right)$ at $12 \mathrm{GHz}$ along a meander loop with $n=5$, where the total line length of the meander is $4\left(5 s_{v}+4 s_{h}\right) \approx 1.336 \lambda_{g}$ with $s_{v}=6 \Delta_{\text {loop }}=$ $0.0303 \lambda_{12}$ and $s_{h}=5 \Delta_{\text {loop }} \approx 0.0253 \lambda_{12}$. The reduction ratio is calculated to be $r_{\text {loop }}=\left(5 s_{v} / s_{\text {str }}\right)^{2} \approx 62 \%$. It is found that the meander loop has a standing wave current, with an almost resistive input impedance (of $52+j 0.3 \Omega$ ). In other words, the meander loop is almost in a resonant state. For confirmation, the current distribution obtained by using the FDTDM [12] is also presented with black and white dots.

Table I shows the computation times for obtaining the current at $12 \mathrm{GHz}$ using the present MoM [(19)] and the conventional MoM [4, Eq. (28) ]. The present MoM is faster than the conventional MoM with a computation time ratio of more than 230 .

As seen from the current distribution of the meander loop, the radiation fields from the currents flowing along the $y$-directed wire element pairs that are symmetric with respect to the $x$ axis add in the $z$ direction, while those from the currents flowing along the $x$-directed wire element pairs that are symmetric with respect to the $x$ axis cancel in the $z$ direction. It follows that the meander loop forms an axial beam, which has the maximum radiation intensity in the $z$ direction. Fig. 6 shows the radiation



Fig. 4. Relationship between the sizes of reference loop and meander loop antennas.

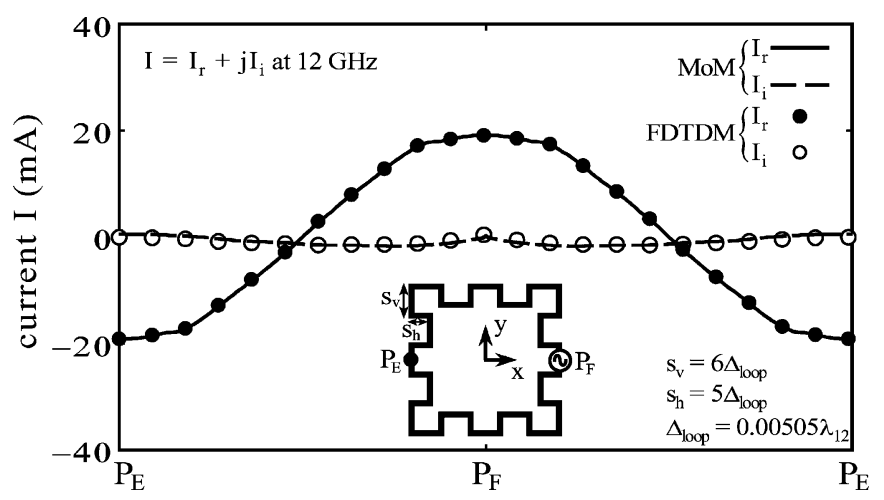

Fig. 5. Current along a meander loop.

pattern of the meander loop, together with that of the reference square loop. The half-power beam width (HPBW) of the meander loop is $99^{\circ}$ in the $x-z$ plane and $85^{\circ}$ in the $y$ - $z$ plane. These are similar to the HPBWs of the reference antenna $\left(93^{\circ}\right.$ in the $x-z$ plane and $83^{\circ}$ in the $y-z$ plane). It follows that the transformation from the straight filaments to the meander filaments does not deteriorate the radiation pattern.

So far, a test frequency of $12 \mathrm{GHz}$ has been used. Next, the frequency response of the meander loop is investigated, using frequencies from 10.5 to $13.5 \mathrm{GHz}$. Fig. 7 shows the voltage standing-wave ratio (VSWR) (relative to $50 \Omega$ ) as a function of frequency. The frequency bandwidth for a VSWR $=2$ criterion is calculated to be $5.2 \%$. Within this $5.2 \%$ VSWR bandwidth, the gain is relatively constant with a maximum value of $5.9 \mathrm{dBi}$. Note that the gain $G$ is calculated using $\left\{\left(\left|E_{\theta} / \sqrt{2}\right|^{2}+\right.\right.$ $\left.\left.\left|E_{\phi} / \sqrt{2}\right|^{2}\right) / Z_{0}\right\} /\left(P_{\text {in }} / 4 \pi r^{2}\right)$, where $E_{\theta}$ and $E_{\phi}$ are the radiation field components expressed using spherical coordinates $(r, \theta, \phi), Z_{0}$ is the intrinsic impedance $120 \pi \Omega$, and $P_{\text {in }}$ is the power input to the antenna. Also, note that the gain of the reference antenna is close to that of the meander loop, showing a maximum value of $6.2 \mathrm{dBi}$ within the same frequency range of 10.5 to $13.5 \mathrm{GHz}$. 
TABLE I

COMPARISON OF COMPUTATION TIMES FOR A MEANDER LOOP ANTENNA USING THE PRESENT MOM AND CONVENTIONAL MOM

\begin{tabular}{|c|c|c|}
\hline \multicolumn{3}{|c|}{ Matrix Size: $200 \times 200$, CPU: Pentium ${ }^{\circledR} 4$, Clock frequency: $1.7 \mathrm{GHz}$, Memory Size: $1 \mathrm{C}$} \\
\hline \multicolumn{3}{|c|}{$\left(\varepsilon_{\mathrm{r}}=2.5, \mathrm{~B}=24 \Delta_{\text {loop }}, \mathrm{s}_{\mathrm{v}}=6 \Delta_{\text {loop }}, \mathrm{s}_{\mathrm{h}}=5 \Delta_{\text {loop }}, \mathrm{n}=5, \Delta_{\text {loop }}=0.00505 \lambda_{12}, \mathrm{f}=12 \mathrm{GHz}\right)$} \\
\hline \multicolumn{2}{|c|}{ Computation Time } & \multirow{2}{*}{$\begin{array}{l}\text { Computation time ratio } \\
\text { (Conventional / Present) }\end{array}$} \\
\hline Present MoM & Conventional MoM & \\
\hline 26 (sec.) & 5998 (sec.) & 231 \\
\hline
\end{tabular}

TABLE II

Comparison of Computation Times for a Grid ARray ANTENNA Using the PRESENT MoM and Conventional MoM

Matrix Size: $1055 \times 1055, \mathrm{CPU}$ : Pentium ${ }^{\circledR} 4$, Clock frequency: $1.7 \mathrm{GHz}$, Memory Size: $1 \mathrm{~GB}$

\begin{tabular}{c|c|c}
\hline \multicolumn{2}{c}{$(\mathrm{N}=5, \mathrm{f}=12 \mathrm{GHz})$} \\
\hline Computation Time & $\begin{array}{c}\text { Computation time ratio } \\
\text { (Conventional / Present) }\end{array}$ \\
\hline $904(\mathrm{sec}$ ) & Conventional MoM & 937 \\
\hline
\end{tabular}



Fig. 6. Radiation patterns of reference loop and meander loop antennas.

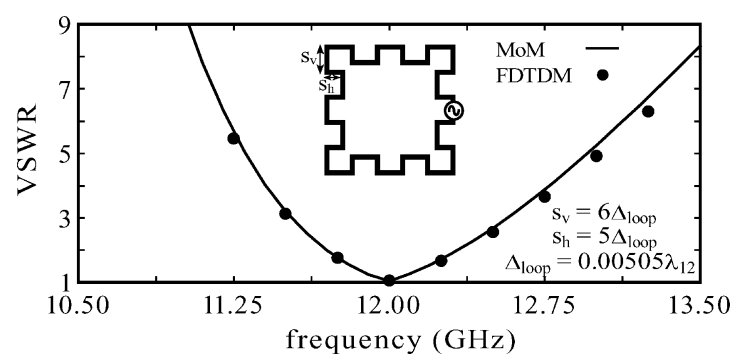

Fig. 7. VSWR of a meander loop antenna.

\section{B. Grid Array Antenna}

Fig. 8 shows a grid array printed on a dielectric substrate of relative permittivity $\varepsilon_{r}$ and thickness $B$. The $x$ - and $y$-directed wire elements of radius $\rho$ have lengths $L_{x}$ and $L_{y}$, respectively. Each $x$-directed element acts as a radiation element and each $y$-directed element acts as a transmission line joining neighboring $x$-directed elements [7]-[11]. The $y$-directed element length $L_{y}$ is always chosen to be $L_{y}=2 L_{x}$ in this paper. The number of $x$-directed elements $=2[1+2+3+\cdots+(N-1)]+N$.

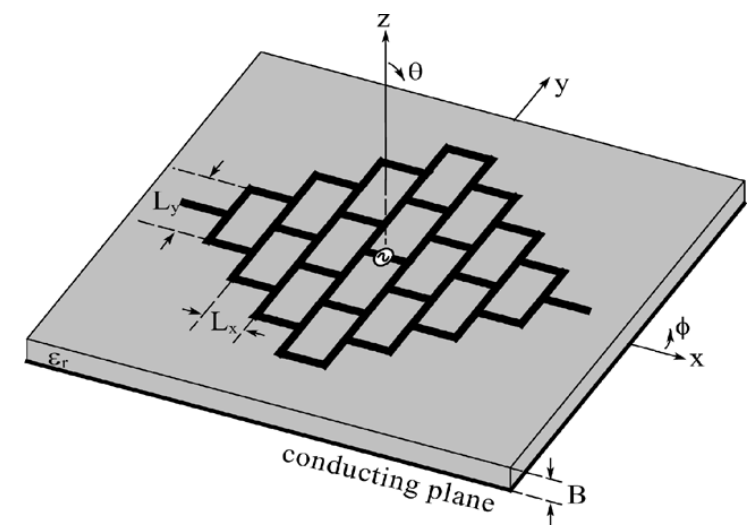

Fig. 8. A grid array antenna.

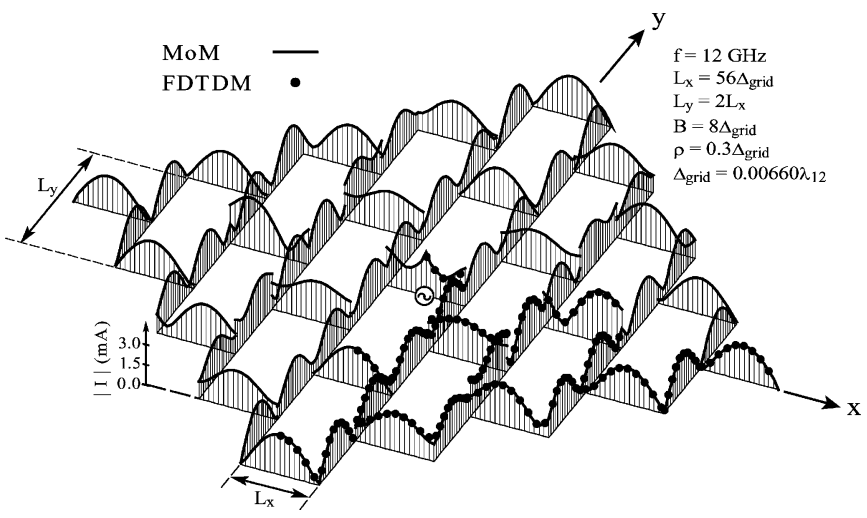

Fig. 9. Current amplitude along a grid array.

The test frequency is set to be $12 \mathrm{GHz}$. The grid array to be analyzed here has the following parameters: $\varepsilon_{r}=2.6, B=$ $8 \Delta_{\text {grid }}=0.0528 \lambda_{12}, \rho=0.3 \Delta_{\text {grid }}=0.00198 \lambda_{12}$, and $N=$ 5 , where $\Delta_{\text {grid }}=0.00660 \lambda_{12} \approx 0.00885 \lambda_{g}$.

Fig. 9 shows the current amplitude $|I|$ at $12 \mathrm{GHz}$, where $L_{x}=56 \Delta_{\text {grid }} \approx 0.496 \lambda_{g}$ and $L_{y}=2 L_{x}=112 \Delta_{\text {grid }} \approx$ $0.993 \lambda_{g}$. Note that $L_{x}$ and $L_{y}$ are approximately $\lambda_{g} / 2$ and $1 \lambda_{g}$, respectively. For confirmation, the current distribution obtained using the FDTDM is presented with black dots. The MoM and FDTDM results are in good agreement. The computation times 


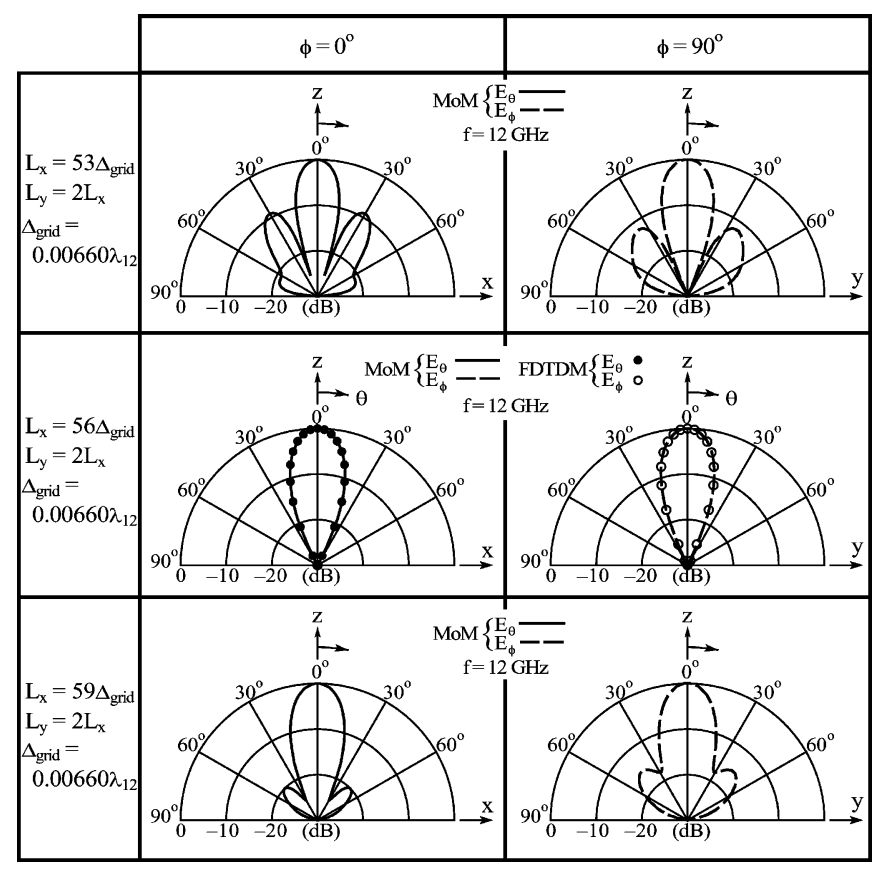

Fig. 10. Radiation pattern of a grid array antenna as a function of the $x$-directed element length $L_{x}$.

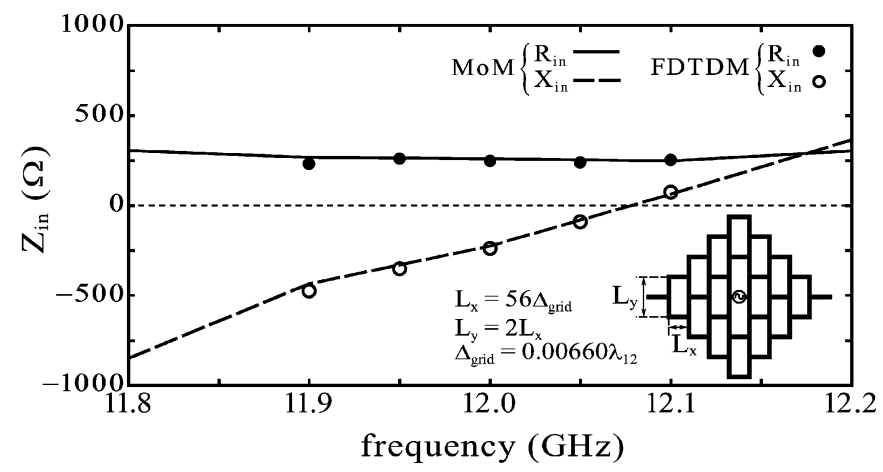

Fig. 11. Input impedance $Z_{\text {in }}$ of a grid array antenna.

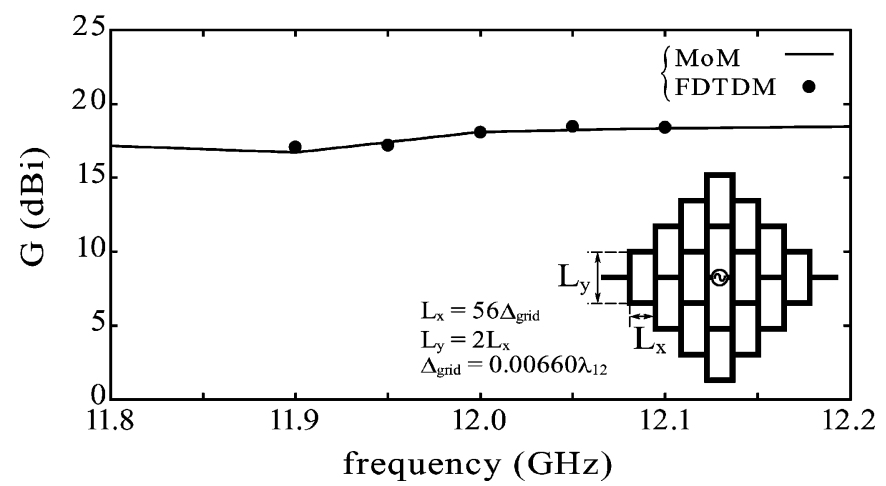

Fig. 12. Gain of a grid array antenna.

for obtaining the current at $12 \mathrm{GHz}$ using the present and conventional MoM methods are shown in Table II. The present MoM reduces the computation time by a factor of 937 .

Fig. 10 shows the radiation patterns at $12 \mathrm{GHz}$ for $L_{x}=$ $56 \Delta_{\text {grid }} \pm 3 \Delta_{\text {grid }} \approx 0.497 \lambda_{g} \pm 0.0266 \lambda_{g}$. The radiation patterns for $L_{x}=56 \Delta_{\text {grid }}$ do not have side lobes in the $x-z$ and $y-z$ planes, as desired. The HPBW of the radiation beam for $L_{x}=$ $56 \Delta_{\text {grid }}$ is $20^{\circ}$ in the $x$ - $z$ plane and $19^{\circ}$ in the $y$ - $z$ planes, with a gain of approximately $18.1 \mathrm{dBi}$.

Figs. 11 and 12 show the frequency responses of the input impedance ( $Z_{\text {in }}=R_{\text {in }}+j X_{\text {in }}$ ) and the gain $G$, respectively. It is found that the resistive value $R_{\text {in }}$ is relatively constant in this analysis range. The gain is between 17.2 and $18.5 \mathrm{dBi}$. The validity of the present MoM results for the gain is confirmed by the FDTDM results.

\section{CONCLUSION}

This paper presents a numerical technique based on the MoM for obtaining the current along an arbitrarily shaped wire printed on a dielectric substrate, where the input impedance matrix element $Z_{m, n}=Z_{m, n}^{C}\left(E_{\psi s}+E_{\psi}\right)+\Delta Z_{m, n}^{C}(\Delta E)$ is formulated using three terms $Z_{m, n}^{\psi s}, Z_{m, n}^{\psi}$, and $\Delta Z_{m, n}^{C}$, involving single-, double-, and triple-integral calculations, respectively.

To show the power of the new formulation of $Z_{m, n}$, two antennas (meander loop and grid array antennas) are analyzed. The computation time for obtaining the current of each antenna based on the new $Z_{m, n}$ is compared with the computation time required using the conventional impedance matrix element, composed of four terms expressed in triple-integral form. It is revealed that the MoM with the new $Z_{m, n}$ drastically reduces the computation time (by a factor of 231 for the meander loop antenna and 937 for the grid array antenna). Thus, the extraction of the single-integral term $Z_{m, n}^{\psi s}$ from the original double-integral $Z_{m, n}^{C}\left(E_{\psi s}+E_{\psi}\right)$ and application of the interpolation technique to the triple-integral term $\Delta Z_{m, n}^{C}$ are found to be effective in reducing the MoM computation time.

The radiation characteristics of these two antennas are also calculated using the obtained currents. The calculations reveal the following facts.

1) A loop antenna with meander cells (which is $62 \%$ smaller than the reference loop without meander cells) shows radiation patterns similar to those of the reference loop. The meander loop has a frequency bandwidth of $5.2 \%$ for a $\mathrm{VSWR}=2$ criterion.

2) A grid array with appropriately selected grid cell lengths $L_{x}$ and $L_{y}$ shows radiation patterns with no side lobes in the principal $x-z$ and $y-z$ planes. A gain of $18.1 \mathrm{dBi}$ is obtained with 25 radiation elements.

\section{ACKNOWLEDGMENT}

The authors thank V. Shkawrytko for his invaluable assistance in the preparation of this paper.

\section{REFERENCES}

[1] I. E. Rana and N. G. Alexopoulos, "Current distribution and input impedance of printed dipoles," IEEE Trans. Antennas Propag., vol. AP-29, pp. 99-105, Jan. 1981.

[2] H. Nakano, S. R. Kerner, and N. G. Alexopoulos, "The moment method solution for printed wire antennas of arbitrary configuration," IEEE Trans. Antennas Propag., vol. 36, pp. 1667-1674, Dec. 1988.

[3] R. F. Harrington, Field Computation by Moment Methods. New York: Macmillan, 1968

[4] H. Nakano, A Numerical Approach to Line Antennas Printed on Dielectric Materials. Amsterdam, The Netherlands: North Holland, 1991, vol. 68, Computer Physics Communications, pp. 441-450. 
[5] H. Nakano, K. Hirose, I. Ohshima, and J. Yamauchi, "An integral equation and its application to spiral antennas on semi-infinite dielectric materials," IEEE Trans. Antennas Propag., vol. 46, pp. 267-274, Feb. 1998.

[6] H. Nakano, T. Kawano, H. Mimaki, and J. Yamauchi, "An analysis of a printed grid array antenna by a fast MoM calculation technique," in Proc. 11th Int. Conf. Antennas Propagation, Manchester, U.K., Apr. 2001, pp. 302-305.

[7] J. D. Kraus, Antennas, 2nd ed. New York: McGraw-Hill, 1988, pp. 491-496.

[8] R. Conti, J. Toth, T. Dowling, and J. Weiss, "The wire grid microstrip antenna," IEEE Trans. Antennas Propag., vol. AP-29, pp. 157-166, Jan. 1981.

[9] H. Nakano, T. Kawano, H. Mimaki, J. Yamauchi, and K. Hirose, "Grid array antennas," in Proc. ICCS'96, Singapore, Nov. 1996, pp. 307-311.

[10] H. Nakano, T. Kawano, and J. Yamauchi, "Meander-line grid-array antenna," Proc. Inst. Elect. Eng. Microwave Antennas Propag., vol. 145, no. 4, pp. 309-312, Aug. 1998 .

[11] E. A. Soliman, S. Brebels, G. Vandenbosch, and E. Beyne, "X-band brick wall antenna fed by CPW," Electron. Lett., vol. 34, no. 9, pp. 836-838, Apr. 1998.

[12] A. Taflove, Computational Electrodynamics: The Finite-Difference Time Domain Method. Norwood, MA: Artech House, 1995.

[13] D. R. Jackson and N. G. Alexopoulos, "An asymptotic extraction technique for evaluating Sommerfeld-type integrals," IEEE Trans. Antennas Propag., vol. 34, pp. 1467-1470, Dec. 1986.

[14] H. Nakano, Analysis Methods for Electromagnetic Wave Problems, E. Yamashita, Ed. Norwood, MA: Artech House, 1996, vol. 2, ch. 3.

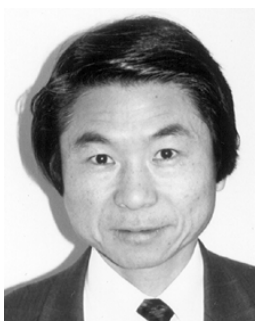

Hisamatsu Nakano (M'75-SM'87-F'92) was born in Ibaraki, Japan, on April 13, 1945. He received the B.E., M.E., and Dr.E. degrees in electrical engineering from Hosei University, Tokyo, Japan, in 1968, 1970, and 1974, respectively.

Since 1973, he has been a Member of the Faculty of Hosei University, where he is now a Professor of Electronic Informatics. His research topics include numerical methods for low- and high-frequency antennas and optical waveguides. He has published more than 200 refereed journal papers, more than 180 international symposium papers, and more than 550 national symposium papers. He is the author of Helical and Spiral Antennas (New York: Wiley, 1987) and the coauthor of Analysis Methods of Electromagnetic Wave Problems, volume 2 (Norwood, MA: Artech House, 1996). One of his most significant contributions is the development of a parabolic reflector antenna using a backfire helical feed for reception of direct broadcast satellite (DBS) TV programs. He has also contributed to the development of two types of small flat DBS antennas using curled and helical elements. He was a Visiting Associate Professor at Syracuse University (March to September 1981) and a Visiting Professor at the University of Manitoba (March to September 1986) and University of California, Los Angeles (September 1986 to March 1987). In 2001, he held a Guest Professorship at Shanghai Jiao Tong University, China. He is an Associate Editor of Electromagnetics and Asian Information-Science-Life.

Prof. Nakano received the Institution of Electrical Engineers International Conference on Antennas and Propagation Best Paper Award and the IEEE Transactions on Antennas and Propagation Best Application Paper Award (H. A. Wheeler Award) in 1989 and 1994, respectively. In 2001, he received the Award of Distinguished Technical Communication from the Society for Technical Communication; and the Science and Technology Progress Award from Hangzhou, China. He is an Associate Editor of IEEE ANTENNAS AND PROPAGATION MAGAZINE and IEEE ANTENNAS AND WIRELESS PROPAGATION LETTERs. He was a past elected member of the IEEE Antennas and Propagation Society Administrative Committee.



Toru Kawano (M'97) was born in Oita, Japan, on December 26, 1971. He received the B.E., M.E., and Dr.E. degrees in electrical engineering from Hosei University, Tokyo, Japan, in 1995, 1997, and 2001, respectively.

Since 2001, he has been an Assistant with the National Defense Academy of Japan, Kanagawa, Japan

Dr. Kawano is a member of the Institute of Electronics, Information and Communication Engineers of Japan

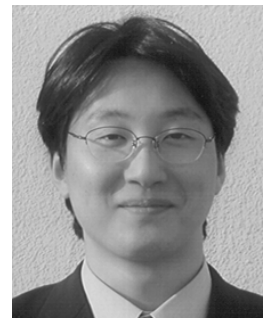

Yousuke Kozono was born in Tochigi, Japan, on February 23, 1979. He received the B.E. and M.E. degrees in electrical engineering from Hosei University, Tokyo, Japan, in 2001 and 2003, respectively.

He has been with Sony Corporation, Tokyo, since 2003.

Mr. Kozono is a member of the Institute of Electronics, Information and Communication Engineers of Japan.

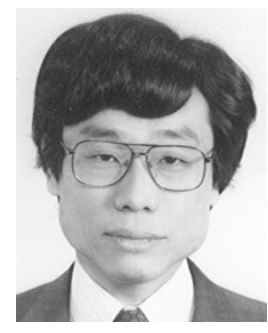

Junji Yamauchi (M'85) was born in Nagoya, Japan, on August 23, 1953. He received the B.E., M.E., and Dr.E. degrees from Hosei University, Tokyo, Japan, in 1976, 1978, and 1982, respectively.

From 1984 to 1988, he was a Lecturer in the Electrical Engineering Department, Tokyo Metropolitan Technical College. Since 1988, he has been a Member of the Faculty of Hosei University, where he is now a Professor of electronic informatics. His research interests include optical waveguides and circularly polarized antennas. $\mathrm{He}$ is the author of Propagating Beam Analysis of Optical Waveguides (Hertfordshire, U.K. Research Studies Press, 2003).

Dr. Yamauchi is a member of the Optical Society of America and the Institute of Electronics, Information and Communication Engineers of Japan. 